\title{
Evaluación de la utilidad de la monitorización continua de la presión arterial mediante la función Haste en los pacientes en hemodiálisis
}

\author{
Montserrat Marcet Duran \\ Concepción Blasco Cabañas \\ Anna Borrell de Puig \\ Manuel García García \\ María Jesús López Parra \\ Thaîs López Alba \\ Nuria Mañé Buixó \\ Carmen Moya Mejía \\ Laura Picazo López \\ Anna Vilas Rivares \\ Elios Yuste Giménez
}

Servicio de Nefrología.

Hospital Parc Taulí.

Sabadell (Barcelona)

\section{RESUMEN}

La hemodiálisis puede generar inestabilidad hemodinámica, desencadenando episodios de hipotensión, algunos sintomáticos. La función HASTE del monitor Colin, permite la monitorización no invasiva y continuada de la TA mediante un algoritmo de cálculo, para el cual precisa la señal del ECG y la onda de saturación de $\mathrm{O}_{2}$.

Con los objetivos de validar la toma de TA mediante el monitor Colin y demostrar la capacidad de detección de hipotensión presintomática mediante la función HASTE, se realiza un estudio descriptivo longitudinal, durante 4 semanas. Para validar el monitor Colin se comparó la TA tomada cada 30 minutos en pacientes en HD con el

\begin{tabular}{|c|}
\hline Correspondencia \\
Montserrat Marcet \\
Servicio de Nefrología \\
Hospital Parc Taulí \\
C/ Parc Taulí s/n \\
08208 Sabadell (Barcelona) \\
\hline
\end{tabular}

monitor y con el método convencional (aparato de tensión automática).

Para demostrar la capacidad de detección de hipotensión presintomática mediante la función HASTE y la disminución de hipotensión sintomática mediante una actuación precoz, elegimos pacientes que presentaban una TA inferior o igual a 100 / 50 mmHg en un $30 \%$ de las HD efectuadas durante los 2 meses previos.

Se hallaron 57 episodios de hipotensión sintomática, 27 episodios en el período convencional y 30 episodios durante el período Colin, sin hallarse diferencias estadísticamente significativas entre ambos métodos. Las hipotensiones sintomáticas durante el período Colin tienen una media TA sistólica $91,03 \mathrm{mmHg}$ y TA diastólica de 51,1 $\mathrm{mmHg}$. Con el método convencional se registraron 237 medidas de TA sistólica inferiores a los límites establecidos, con una media de 89,09 mmHg y 87 medidas de TA diastólica con una media de $45,36 \mathrm{mmHg}$. 
Por tanto, no hay diferencia estadística significativa en la toma de la TA entre ambos aparatos y se observan más número de registros de TA por debajo de los límites establecidos en el período Colin por la monitorización continua a través de la función HASTE. Las hipotensiones sintomáticas registradas durante el período convencional son más acusadas requiriendo un mayor número de intervenciones de enfermería.

$\begin{array}{ll}\text { PALABRAS CLAVE } & \text { HEMODIÁLISIS } \\ & \text { HIPOTENSIÓN } \\ & \text { FUNCIÓN HASTE } \\ & \text { MONITORIZACIÓN CONTÍNUA NO INVASIVA } \\ & \text { DETECCIÓN PRECOZ }\end{array}$

\section{EVALUATION OF THE CONTINUOUS MONITO- RING OF BLOOD PRESSURE BY THE HASTE FUNCTION IN PATIENTS UNDER HAEMODYALI- SIS}

HAEMODYALISIS can produce episodes of haemodinamic instability leading to symptomatic episodes of hypotension. The HASTE function of the Colin monitor allows the non-invasive monitoring of blood preassure using an algorithm that needs a precise ECG signal and the $\mathrm{O}_{2}$ saturation wave.

To validate the measurements of blood preasure using the Colin monitor and to demonstrate its capacity to detect pre-symptomatic hypotension using the HASTE function, we performed a longitudinal descriptive study over 4 weeks. To validate the Colin monitor we compared the blood pressure measured every 30 minutes with the monitor and with a conventional method.

To demonstrate the capacity of detection of presymptomatic hypotension, we selected a group of patients with blood pressure equal or inferior to $100 / 50 \mathrm{mmHg}(30 \%$ of patients in the previous two months).

We detected 57 episodes of symptomatic hypotension (27 in the conventional period and 30 during the Colin period). There were no stastistically significant differences.
In conclusion, there are no statistically significant differences in the blood pressure measured by the two methods. The number of registers of blood pressure during the Colin period is under the thresold when the HASTE function is used. Symptomatic hypotensions during the conventional period are more pronounced and require more interventions from the nursing team.

$\begin{array}{ll}\text { KEY WORDS: } & \text { HAEMODYALISIS } \\ & \text { HYPOTENSION } \\ & \text { HASTE FUNCTION } \\ & \text { CONTINOUS NON-INVASIVE MONOTORIZATION } \\ & \text { EARLY DETECTION }\end{array}$

\section{INTRODUCCIÓN}

La hemodiálisis (HD) puede generar cierta inestabilidad hemodinámica, desencadenando episodios de hipotensión arterial, a menudo sintomáticos, en un cierto número de pacientes de especial predisposición ${ }^{(1,2)}$. Estos episodios de hipotensión son de relevancia no sólo por la repercusión clínica para el paciente, con aparición de sintomatología (náuseas, vómitos, calambres a nivel de EEII e incluso pérdida de conciencia), sino también por la posibilidad de complicaciones derivadas de la disminución de la perfusión tisular, pudiendo desencadenar episodios de ángor pectoris, ángor intestinal e incluso hipoperfusión cerebral ${ }^{(3,4)}$.

Con el propósito de minimizar estos episodios, así como para un correcto control clínico del paciente, durante las sesiones de HD se requiere una estricta monitorización, tanto de la presencia de eventos clínicos como de los parámetros hemodinámicos, mediante la toma de tensión arterial (TA) de manera reglada e intermitente por parte del equipo de enfermería ${ }^{(5,6)}$. A pesar de este control tan intenso de la TA durante la sessión de HD, pueden aparecer episodios hipotensivos clínicamente relevantes en los intervalos entre las tomas de TA. Estos episodios generan, además de problemas añadidos para el paciente, la necesidad de una actuación urgente tanto por el personal médico como por el equipo de enfermería.

Por todo ello, resultaría útil disponer de un método continuo no invasivo para la monitorización de la TA que permitiera, mediante un mecanismo de alarma, detectar 
las caídas de la TA en fases presintomáticas, facilitando una actuación precoz para impedir o minimizar la aparición de síntomas.

La función HASTE del monitor Colin BP-88 Si NEXT permite precisamente esta monitorización no invasiva de manera continuada de la TA mediante la estimación de la misma, a través de un cálculo algoritmo que realiza el mismo monitor. Esta función ha sido validada en pacientes postquirúrgicos y en pacientes críticos, comparándola con la monitorización convencional utilizada en estas unidades (aparato de medición automático no invasivo) y con la monitorización invasiva de la TA, siendo considerado un método de utilidad en estos pacientes, tanto para la detección de hipotensiones como de hipertensión arterial ${ }^{(7,8,9,10,11)}$.

\section{OBJETIVOS DEL ESTUDIO}

1) Validar la toma de la tensión arterial mediante el monitor Colin.

2) Demostrar la capacidad de detección de hipotensión presintomática mediante la función HASTE y observar la disminución de hipotensión sintomática mediante una actuación precoz.

\section{MATERIAL Y MÉTODOS}

Realizamos un estudio descriptivo longitudinal utilizando como prueba estadística la media. Usamos el monitor Colin BP-88 Si NEXT que permite registrar simultaneamente las ondas de electrocardiograma (ECG), la saturación de oxígeno y el registro de $\mathrm{TA}$, disponiendo además de la función HASTE. Esta función se utiliza para detectar una caída de la TA del paciente, activandóse la alarma para el rango de TA que se haya prefijado en el monitor.

Para detectar esta probable disminución de la TA, el monitor realiza un cálculo alogarítmiico en función de las alteraciones del ECG y/o de la saturación de $\mathrm{O} 2$ del paciente. Así pues, para que el monitor Colin pueda aplicar la función HASTE es preciso que el paciente lleve durante toda la HD:

- Electrodos para el registro de ECG

- Sensor de saturación de $\mathrm{O}_{2}$

- Manguito de TA

Para validar la toma de tensión arterial mediante el monitor Colin comparamos las cifras de TA del monitor
Colin y las cifras de TA del aparato de medición de TA usado en nuestra unidad (aparato de medición automático no invasivo); para esto escogimos a 21 pacientes en programa de HD ambulatoria controlados en nuestro centro. Se excluyeron aquellos pacientes con patología asociada de mal pronóstico (enfermedades neoplásicas malignas) o con alguna enfermedad intercurrente durante el período de estudio que fue de 4 semanas. Para la recogida de datos, a cada paciente del grupo se le monitorizó la TA durante toda la $\mathrm{HD}$, usando simultáneamente el monitor Colin y el método convencional usado en nuestra unidad. Se registraron las cifras de TA a intervalos de 30 minutos, desde inmediatamente antes de la conexión hasta después de la desconexión.

Para el objetivo 2 escogimos otro grupo de pacientes de nuestra unidad de HD. Esta vez el número de pacientes fue de 23 y el período de estudio fue también de 4 semanas. Este grupo de enfermos fue escogido por que habían presentado cifras de TA intra-diálisis inferior o igual a 100/50 mmHg en al menos un 30\% de las últimas HD efectuadas durante los 2 meses previos al estudio (estas cifras no estaban relacionadas con variaciones en su peso seco o por inestabilidad clínica). Se excluyeron nuevamente aquellos pacientes que presentaban alguna patología de mal pronóstico (enfermedades neoplásicas), enfermedades intercurrentes en el momento del estudio, pacientes portadores de marcapasos o aquellos que presentaban saturaciones de oxígeno inferiores al $90 \%$, que pudieran interferir en la correcta integración de datos de la función HASTE.

A cada paciente del grupo de estudio se le monitorizó durante 6 sesiones de HD consecutivas (2 semanas) con el monitor Colin al que se le había activado la función HASTE, con la instauración de alarmas para detección de TA inferior o igual a 100/50 $\mathrm{mmHg}$. Se registraron los valores de TA desde inmediatamente antes de la conexión hasta después de la desconexión a intervalos prefijados de 30 minutos; igualmente se registró la TA cada vez que lo requería la situación clínica del paciente.

A estos mismos pacientes, y durante 6 sesiones más de $\mathrm{HD}$, se les monitorizó la TA pero esta vez usando nuestro método convencional de medición de TA. Se registraron los valores igualmente a intervalos de 30 minutos y cada vez que la situación clínica del paciente lo requería. En ambos períodos de estudio se registró la presencia de eventos clínicos intra-diálisis y su relación con el tiempo, haciendo hincapié en la presencia y severidad de los episodios de hipotensión sintomática. 
Para consensuar la actuación de todo el equipo de enfermería ante la presencia de hipotensión arterial, se elaboró un protocolo de intervención con el fin de homogeneizar la interpretación de los resultados y siempre que la situación clínica del paciente lo permitiera. Así el protocolo ante hipotensiones fue el siguiente:

- Ante TA 90-95 mmHg = $10 \mathrm{cc} \mathrm{Cl} \mathrm{Na}$ al 20\%.

- Ante TA 85- 89 = 100 cc SF + 10 cc Cl Na al 20\%.

- Ante TA inferior a 85 = reducción de la ultrafiltración +100 cc SF + 10 cc Cl Na al 20\%.

Este protocolo sólo se aplicaba a aquellos pacientes incluidos en el segundo objetivo. Así mismo se anotó la necesidad de realización de medidas de actuación extras dependiendo de la necesidad clínica del paciente.

\section{RESULTADOS}

En relación con el primer objetivo no obtuvimos diferencias importantes en cuanto a las mediciones de la TA con ambos aparatos, existiendo un margen de error entre el monitor Colin y nuestro monitor convencional de $1.87 \mathrm{mmHg}$ de media en la TA sistólica y $0,32 \mathrm{mmHg}$ en la TA diastólica.

En referencia al segundo objetivo, se hallaron un total de 57 episodios de hipotensión sintomática, observándose 27 episodios en el período convencional y 30 episodios durante el período de estudio de Colin, sin hallarse diferencias estadísticamente significativas entre ambos grupos. La sintomatología expresada por los pacientes durante las hipotensiones fue de mayor a menor frecuencia: mareos, cefalea, calor, malestar, diaforesis, contracturas, dolor, náuseas y taquicardia. No se registró ningún episodio de hipotensión con clínica severa (pérdida de conciencia).

En cuanto a la valoración de las hipotensiones asintomáticas en las que enfermería aplicó el protocolo establecido para las hipotensiones, observamos 69 intervenciones de enfermería en el período Colin y 90 intervenciones en el período de estudio con el monitor convencional.

A lo largo del período de estudio con monitor Colin se detectaron 316 alarmas de TA sistólica con una media de $89,96 \mathrm{mmHg}$, y 239 alarmas de TA diastólica con una media de 44,49 mmHg. Los márgenes de alarma se establecieron para la TA sistólica en $100 \mathrm{mmHg}$ y para la TA diastólica en $50 \mathrm{mmHg}$ (para tres pacientes se establecieron márgenes diferentes según sus caracte- rísticas especiales). La función HASTE debía activarse inmediatamente por debajo de estos límites, el promedio de margen de error que detectamos fue:

- Para la TA sistólica $9.53 \mathrm{mmHg}$

- Para la TA diastólica $4.92 \mathrm{mmHg}$

Las hipotensiones sintomáticas durante el período Colin tuvieron una media de TA sistólica $91,03 \mathrm{mmHg}$ y TA diastólica de 51,1 $\mathrm{mmHg}$.

En cuanto al control de TA con el método convencional se registraron un total de 237 medidas de TA sistólica inferiores a los límites establecidos, con una media de 89,09 mmHg y 87 medidas de tensión arterial diastólica inferiores a los límites establecidos con una media de $45,36 \mathrm{mmHg}$. Las hipotensiones con clínica durante el período convencional tuvieron una media de TA sistólica $80,88 \mathrm{mmHg}$ y TA diastólica de $55,35 \mathrm{mmHg}$.

El elevado número de alarmas y con ello la activación de la función HASTE fue debida en algunas ocasiones a un mal registro de la onda de ECG (relacionados con el movimiento del paciente) o a una mala detección de la saturación de $\mathrm{O}_{2}$.

Hemos de destacar que durante esta etapa del proceso de investigación 6 pacientes abandonaron el estudio por diferentes motivos: molestias con el manguito, medidas muy continuadas, nerviosismo por todo el aparataje, cambio de opinión respecto a la continuidad del estudio y dolor articular crónico en la extremidad portadora del manguito.

Durante el estudio se detectó que dos de los pacientes en realidad eran hipertensos y que eventualmente presentaban hipotensiones bruscas; fue necesario ajustar el peso teórico e iniciar tratamiento hipotensor los días de no diálisis. En 3 pacientes incluidos en el estudio no se detectó ningún episodio de hipotensión, así mismo no se detectó ningún episodio de hipotensión brusca con pérdida de conciencia.

\section{DISCUSIÓN}

La muestra estudiada es pequeña debido a que en nuestro centro un $80 \%$ de los pacientes son hipertensos. Además, se presentaron pocos episodios de hipotensión sintomática posiblemente debido a la vigilancia y actuación exhaustiva sobre los pacientes por parte de los profesionales de enfermería, acompañada de una actuación precoz durante el período de estudio, demostrando así, que 
el control de los pacientes durante la HD por parte de enfermería es fundamental para evitar complicaciones más severas. Todo ello conlleva que el número de episodios de hipotensión clínicamente relevante sea insuficiente para poder comparar ambos métodos de medición.

Las hipotensiones sintomáticas en el período convencional presentaron cifras más bajas por lo que resultó más difícil de remontar lo que explicaría el elevado número de actuaciones de enfermería registradas durante este período. Así mismo, el número de alarmas fue mayor en el período de Colin seguramente debido a un mal registro de los parámetros hemodinámicos usados en el monitor HASTE y por lo tanto no siempre estaban relacionadas con un caída de la TA del paciente. Hemos de destacar que algunos pacientes expresaron algunas molestias relacionadas con el aparataje usado en el monitor Colin por lo que la muestra de estudio se redujo significativamente.

\section{CONCLUSIONES}

1) No hallamos diferencias estadísticas significativas en la toma de la TA con ambos aparatos.

2) Se observaron más registros de TA por debajo de los límites establecidos en el período Colin por la monitorización continua que permite la función HASTE.

3) Las hipotensiones sintomáticas registradas durante el período convencional fueron más acusadas requiriendo un mayor número de intervenciones de enfermería.

4) La intensificación del control y la detección precoz de la hipotensión son los principales factores implicados en la reducción del número y de la severidad de los episodios de hipotensión sintomática.

5) El papel de los profesionales de enfermería para el control de los pacientes inestables en HD es imprescindible y nunca puede ser sustituido por ningún aparato.

\section{BIBLIOGRAFÍA}

1. Wolkotte C, Hassell DR, Moret K, Gerlag PG, van den Wall Bake AW, van der Sande FM, Kooman JP.
Blood volume control by biofeedback and dialysis-induced symptomatology. A short-term clinical study. Nephron 2002; 92(3):605-9.

2. Straver B, De Vries PM, Donker AJ, ter Wee PM. The effect of profiled hemodialysis on intradialytic hemodynamics when a proper sodium balance is applied. Blood Purif 2002; 20(4):364-9.

3. Begin V, Deziel C, Madore. Biofeedback regulation of ultrafiltration and dialysate conductivity for the prevention of hypotension during hemodialysis. F ASAIO J 2002 May-Jun; 48(3):312-5.

4. Andrulli S, Colzani S, Mascia F, Lucchi L, Stipo L, Bigi MC, Crepaldi M, Redaelli B, Albertazzi A, Locatelli $\mathrm{F}$. The role of blood volume reduction in the genesis of intradialytic hypotension. Am J Kidney Dis 2002 Dec; 40(6): 1244-54.

5. Kyriazis J, Glotsos J, Bilirakis L, Smirnioudis N, Tripolitou M, Georgiakodis F, Grimani I. Dialysate calcium profiling during hemodialysis: use and clinical implications. Kidney Int 2002 Jan; 61(1):276-87.

6. Hoeben H, Abu-Alfa AK, Mahnensmith R, Perazella MA. Hemodynamics in patients with intradialytic hypotension treated with cool dialysate or midodrine. Am J Kidney Dis 2002 Jan; 39(1):102-7.

7. Furuyashiki T, Eguchi E, Nagai S. Examination of effectiveness of a blood pressure monitor in post peration ward management. Harmonized Alert Sensing Technology (HASTE). Expert Nurse 2002 April; 18 (4).

8. Ito H, Komatsu T, Higashida A, Kurimoto N, Wakao M. Evaluation of new algorithms to estimate continuous systolic blood pressure using ECG and pulse oximeter in patients with hemodynamic changes in intensive care unit. Anesthesiology 2001; 95: A582

9. Hantler C, Martin-Bredahl K, Ratnaraj J, Noorily S, Chen J. Non-Invasive continuous detection of hemodynamic changes. Anesthesiology 2001; 95: A543.

10. Hantler C, Chen J, Ratnaraj J, Martin-Bredahl K, Noorily S. Estimates of pulse wave velocity accurately predict continuous non-invasive blood pressure. Anesthesiology 2001; 95: A581.

11. Botey A, Revert L. Hipertensión arterial. En: Farreras $\mathrm{P}$, Rozman $\mathrm{C}$. Medicina Interna. 13 $3^{\mathrm{a}} \mathrm{Ed}$. Madrid: Mosby/Doyma; 1995. pa 667-684. 